\title{
Papillitis in Neurosyphilis
}

\author{
Macovei Mioara-Laura, Georgescu Raluca-Diana \\ Ophthalmology Department, “Dr. Carol Davila” Central Military \\ Emergency University Hospital, Bucharest, Romania
}

Correspondence to: Georgescu Raluca-Diana, MD,

Ophthalmology Department , "Dr. Carol Davila" Central Military Emergency University Hospital,

Bucharest, Romania,

134 Plevnei Street, District 1, Bucharest, Romania,

Mobile phone: +40745 059 442, E-mail: ralucad.georgescu@yahoo.com

Accepted: September 19th, 2019

\begin{abstract}
We present a case of a 47-year-old female patient, with papillitis in the right eye and anterior uveitis in both eyes, as a manifestation of untreated neurosyphilis.

Keywords: papillitis, optic nerve pathology, visual field defects, anterior uveitis
\end{abstract}

\section{Introduction}

Syphilis is an infectious disease caused by Trepona pallidum [1]. This pathology was called "the great imitator" because it may cause symptoms similar to other diseases [2]. It has 3 stages: primary, secondary, tertiary. Each stage has its own clinical signs and symptoms: primary syphilis - chancre, secondary syphilis - macular papular rash, lymphadenopathy, mucosal ulceration, tertiary syphilis - gummas, cardiac and neurological symptoms. There is also latent syphilis with no clinical manifestations but detectable by serological tests. The bacterium can affect the central nervous system and result in neurosyphilis, which can occur at any stage of the disease. If the disease is left untreated, it has a mortality rate of $8 \%$ to $58 \%[1,2]$.

\section{Case report}

We present the case of a 47-year-old female patient, who came in our hospital complaining of sudden and severe decrease in visual acuity in the right eye for two weeks, accompanied by headache and moderate continuous pain in the right eye. For the medical history, we could mention medically controlled hypertension and a neglected hyperthyroidism. In addition, the patient had a history of penicillin allergy.

At presentation, her best-corrected visual acuity was RE counting fingers (CFs), LE: 1 . The IOP was normal in both eyes, BE $16 \mathrm{mmHg}$ on non-contact tonometry.

Slit-lamp examination of the anterior segment revealed multiple small endothelial precipitates in both eyes (Fig. 1) and a Relative Afferent Pupillary Defect (RAPD) present in the right eye. The examination of the posterior pole showed hyperemic optic disc with blurred margins and macular pigmentary abnormalities in the right eye (Fig. 2), drusen along the vascular arcades and in the macular region in the left eye (Fig. 3). 

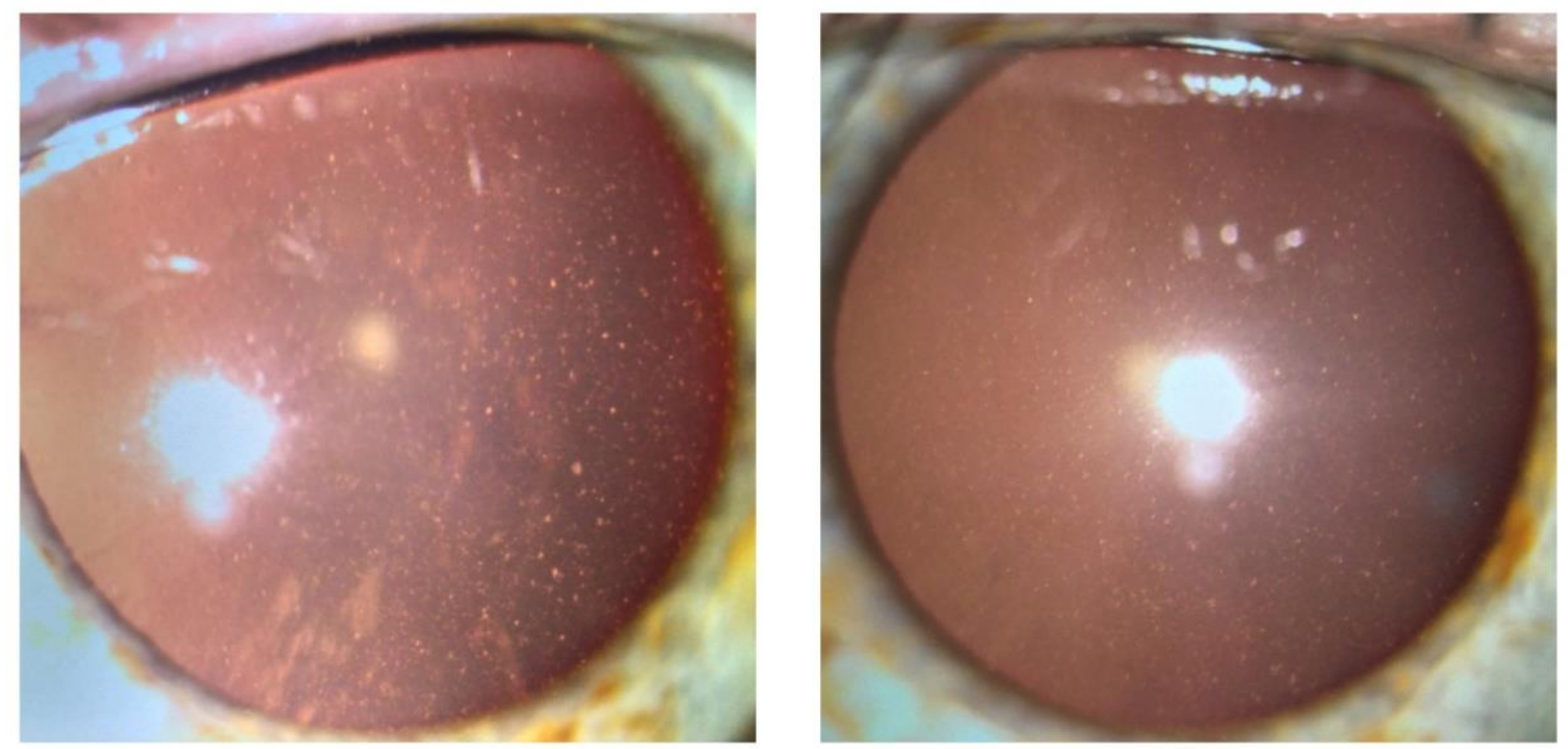

Fig. 1 Multiple, small endothelial precipitates in both eyes

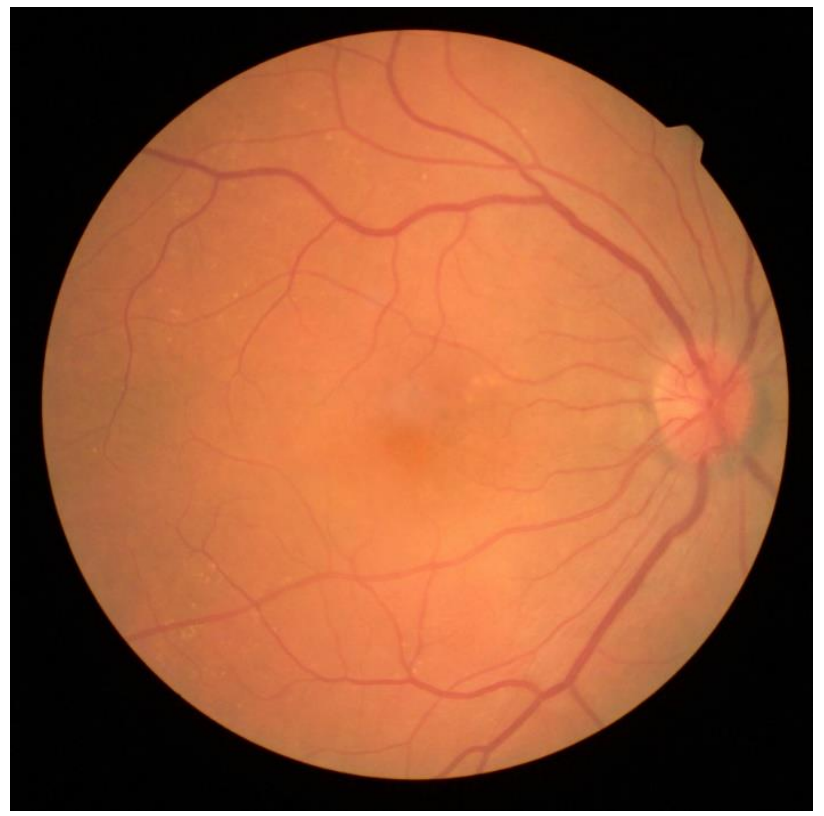

Fig. 2 Hyperemic optic disc with blurred margins and macular pigmentary abnormalities in the right eye

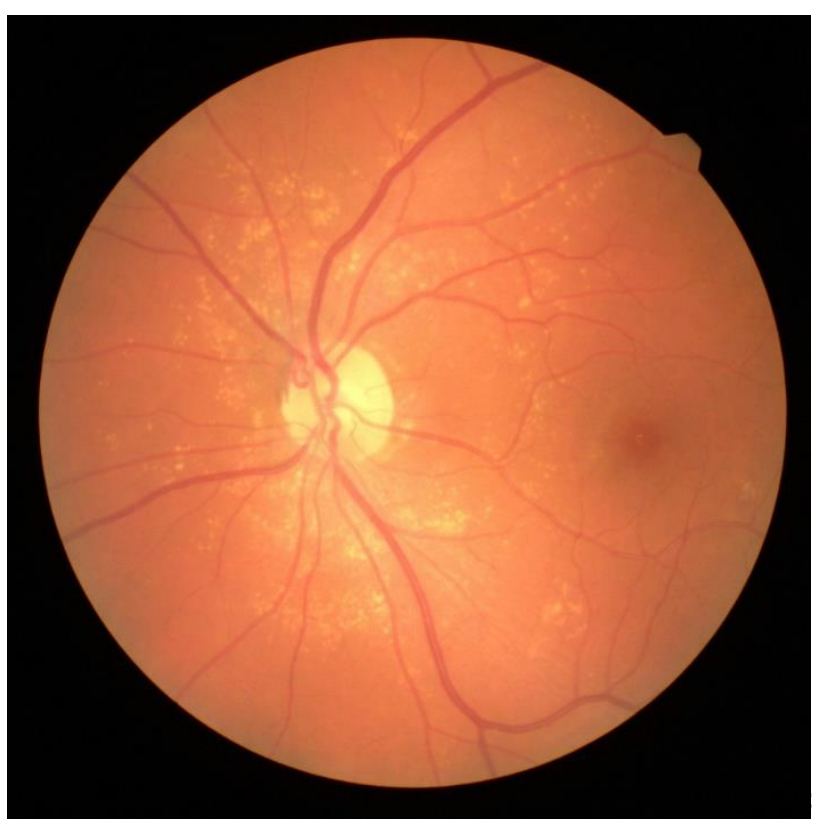

in the right eye. 
using SITA-Standard software showed and multiple non-systematized defects in all temporal hemianopsia in the right eye (Fig. 4) the four quadrants in the left eye (Fig. 5).
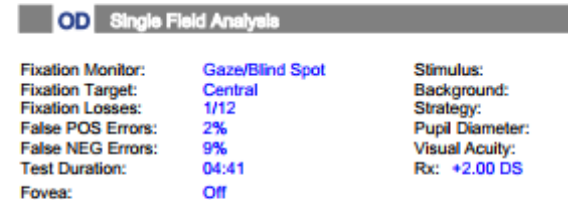

Fovea:

04:41

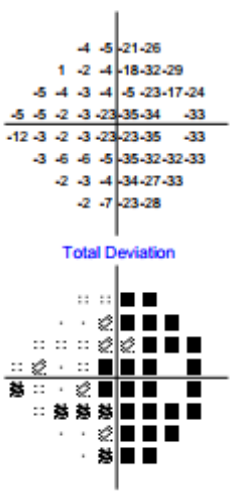

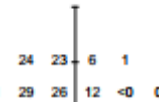

$\begin{array}{llllllll}25 & 28 & 29 & 28 & 27 & 9 & 13\end{array}$

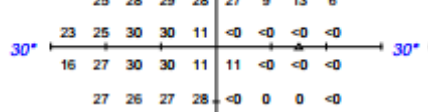

\begin{tabular}{lll|llll}
29 & 29 & 28 & 00 & 4 & 00
\end{tabular}

\begin{tabular}{ll|ll}
28 & 23 & 7 & 3
\end{tabular}

(1)

$-1 \cdot 3 \cdot-19-24$

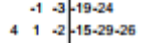

$\begin{array}{cccccc}-2 & -1 & 0 & -2 & -2 \cdot-20-15-22\end{array}$

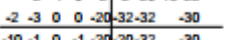

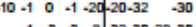

Pattern Deviation

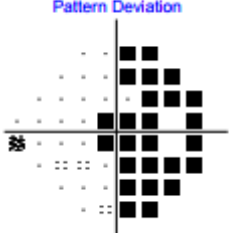

GHT: Oulside Normal Limits

Contral 242 Throchold Toet

III, White Date: Feb 01, 2019

31.5 asb
SITA Fast

Time: $1: 45 \mathrm{PM}$

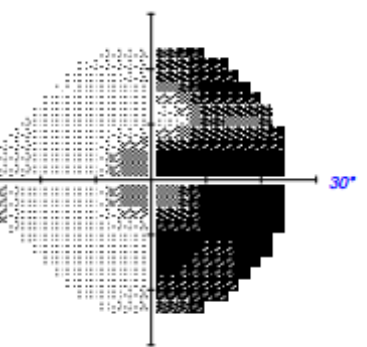

VFI: $\quad 50 \%$

$\begin{array}{ll}\text { MD24-2: } & -14.35 d B P<0.5 \% \\ \text { PSD24-2: } & 13.35 d B P<0.5 \%\end{array}$
Fig. $\quad 4 \quad$ Temporal hemianopsia in the right eye

Fig. 5 Multiple nonsystematized defects in all the four quadrants in the left eye

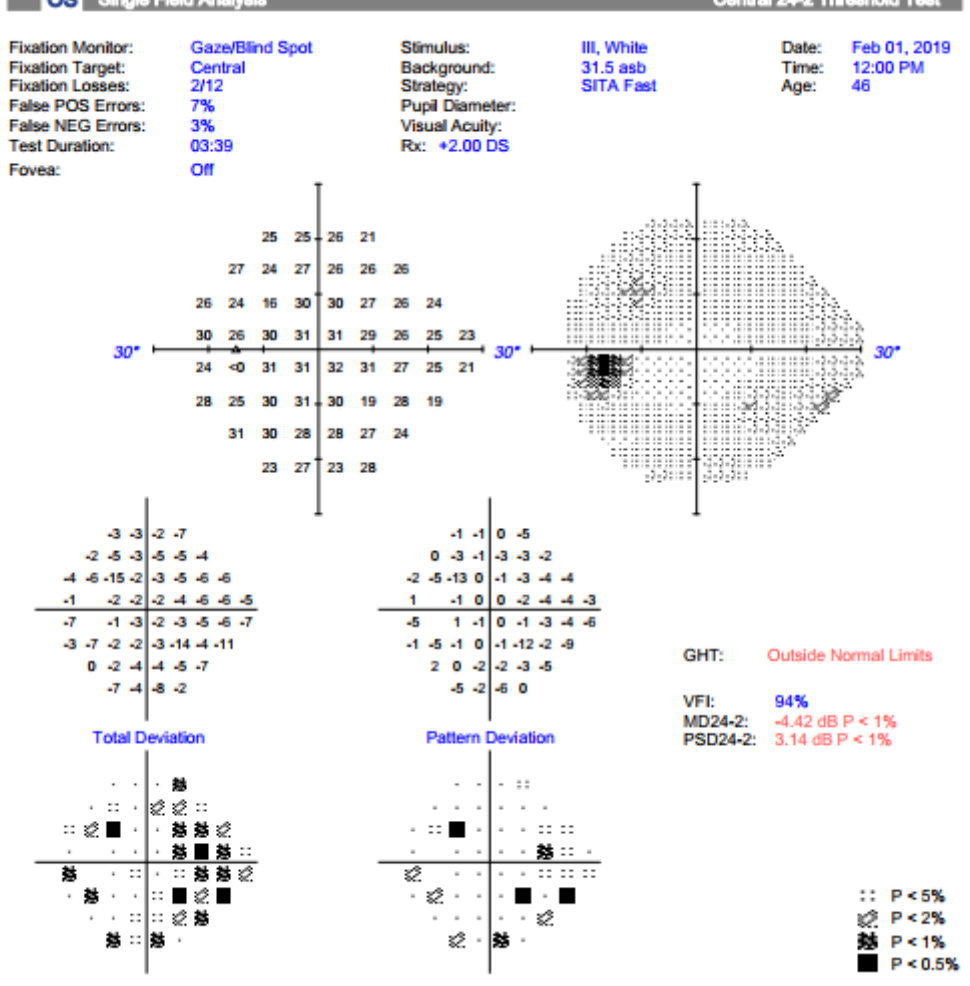


On clinical examination, the patient had plaques $>10 \mathrm{~mm}$ with hemorrhagic crusts on the body, neck, and head (Fig. 6).
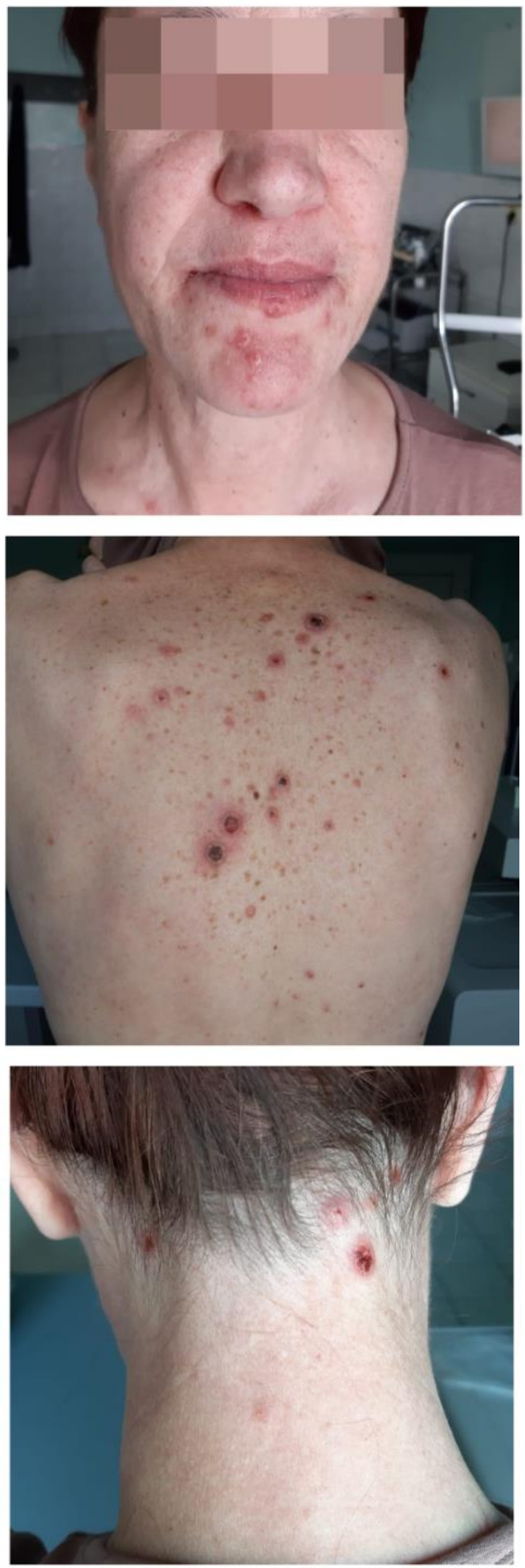

Fig. 6 Plaques $>10 \mathrm{~mm}$ with hemorrhagic crusts
Further blood tests were ordered: complete count blood (CBC), a metabolic panel and a lipid panel, rheumatoid factor, ANA, ANCA, and serum ACE, HLA B27, IgM and IgG for Toxoplasma gondii. The next analyses were raised: fibrinogen - 617.0 mg/ dl (276.00-417.00), erythrocyte sedimentation rate (ESR) - $40 \mathrm{~mm} / 1 \mathrm{~h}$ (1.00$25.00)$, C reactive protein $-28.68 \mathrm{mg} / \mathrm{l}$ (0.005.00 ) and blood glucose - $303 \mathrm{mg} / \mathrm{dl}$ (74.00106.00). Her viral serology was negative for HIV, hepatitis, herpes simplex. TPHA and VDRL were positive.

The otorhinolaryngology and neurological exam were normal and the head and orbit MRI with i.v. contrast was also normal. The diagnoses of type II diabetes mellitus and hyperthyroidism was established by the endocrinological exam and the patient received adequate treatment.

The dermatologic consult suspected lymphomatoid papulosis and tertiary syphilis, but the skin biopsy necessary for the diagnosis of lymphomatoid papulosis was postponed because of the positive test results for Syphilis.

A lumbar puncture was performed with a normal opening pressure. Cerebrospinal fluid protein and glucose were both raised and TPHA and VDRL were positive. The patient was diagnosed with neurosyphilis.

The patient received alternative treatment with $200 \mathrm{mg}$ doxycycline p.o and $2 \mathrm{~g}$ ceftriaxone i.v 14 days, because of her penicillin allergy.

After 7 days of treatment her bestcorrected visual acuity was RE: $0,4 \mathrm{nc}$, LE: 1 . The visual field examination in the right eye showed that the defect from presentation decreased and the defects in the left eye disappeared.

The next follow-up was after one month and her BCVA was BE: 1 and the visual fields in both eyes were within normal limits (Fig. 7). The endothelial precipitates disappeared and the aspect of the optic disc in the right eye was normal (Fig. 8). 

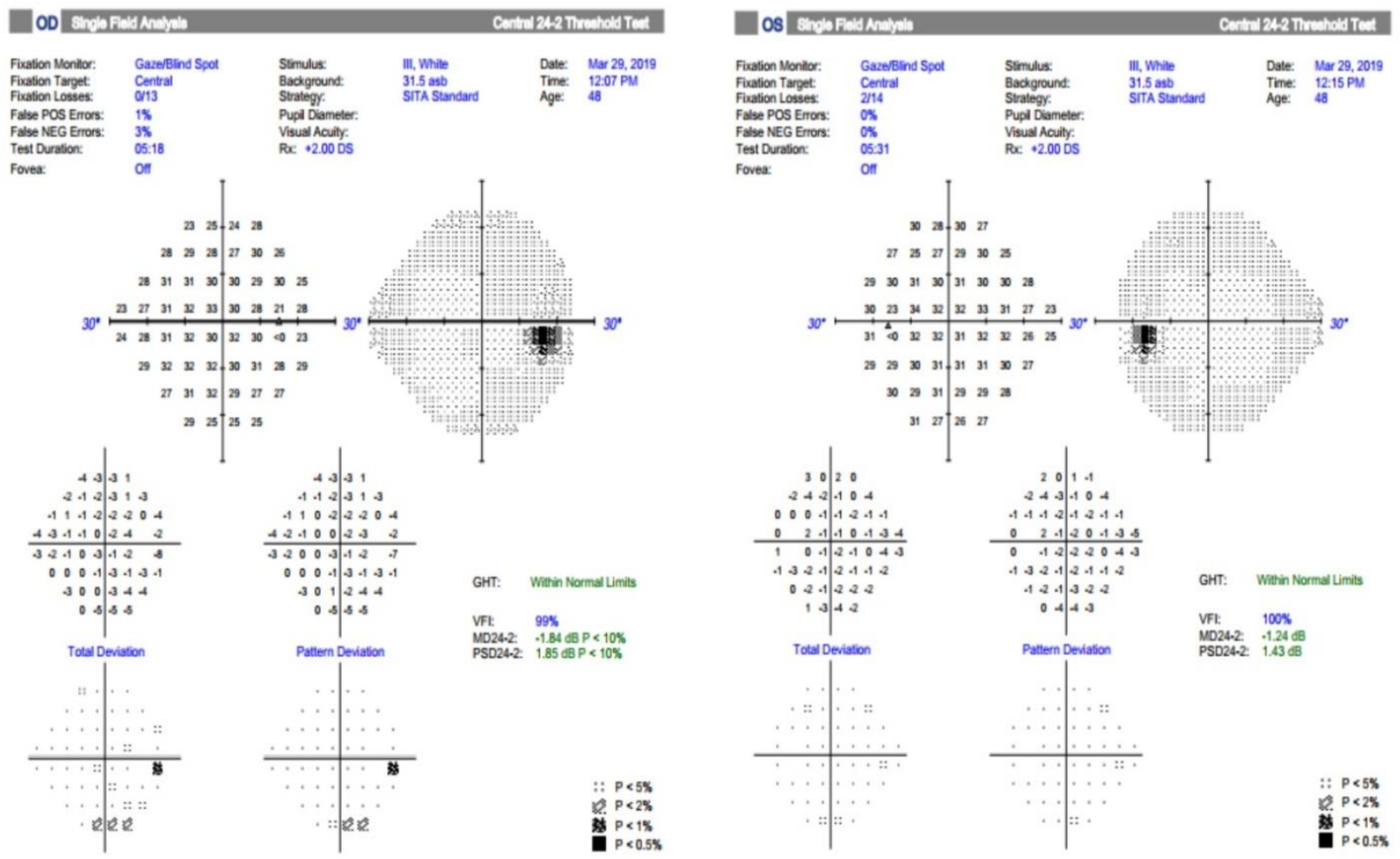

Fig. 7 Visual fields in both eyes were within normal limits after one month

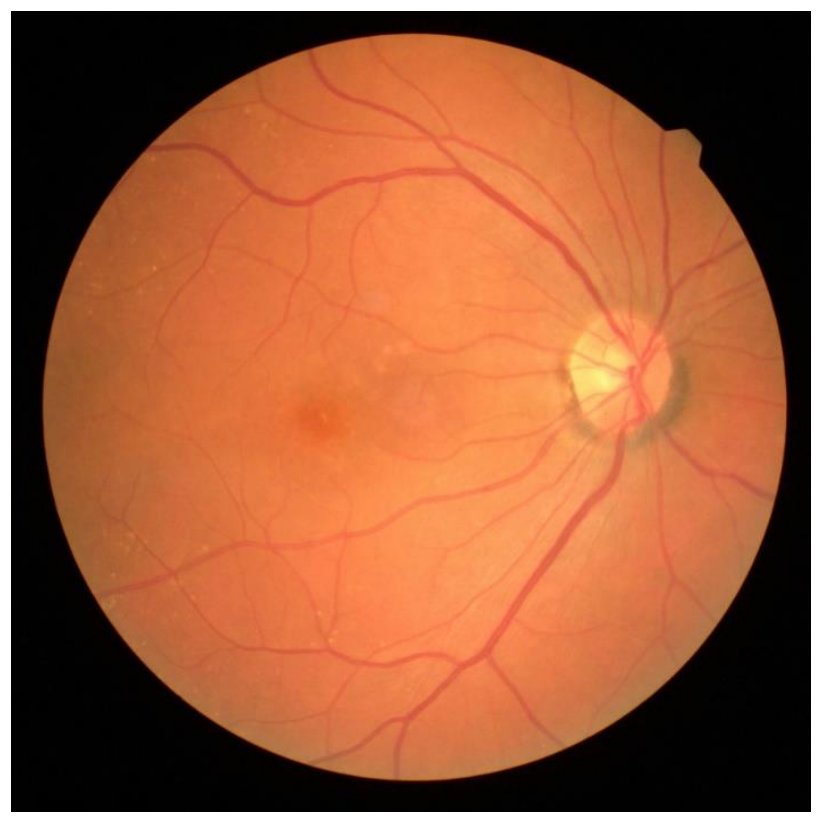

Fig. 8 Normal aspect of the optic disc after treatment

\section{Discussion}

Papillitis or optic neuritis is the inflammation and deterioration of the anterior portion of the optic nerve known as the optic disc [3-5]. The diffuse margins of the optic disc suggested papillitis, papilledema, or anterior ischemic optic neuropathy: papilledema was excluded due to normal opening pressure of the CSF. Some exclusion factors for arteritic AION were age $<70$ years old, absence of jaw claudication, absence of the pale (chalky) aspect of the optic disc and for nonarteritic AION were age $<60$ years old, absence of a crowded disc, and the pain that accompanied the visual loss [7].

Papillitis has many causes including multiple sclerosis, viral or bacterial infections, nutritional or metabolic disorders such as diabetes mellitus and hyperthyroidism [6]. The diagnostic of syphilitic papillitis was performed based on serological positive test, clinical ocular manifestations and the examination and culture of the cerebrospinal fluid. In medical literature, 
only a few cases of syphilitic papillitis were described in immunocompetent patients.

Anterior uveitis is a more common ocular manifestation than papillitis in syphilis. Anterior uveitis occurs in about $4 \%$ of the patients with secondary syphilis; it may be granulomatous or non-granulomatous and is bilateral in $50 \%$ of the cases [6].

The alternative treatment in patients with neurosyphilis and allergy to penicillin is with ceftriaxone $2 \mathrm{~g}$ i.v. for 10-14 days and with doxycycline $200 \mathrm{mg}$ p.o twice daily for 28 days $[8,9]$.

\section{References}

1. Syphilis. CDC. 4 June 2015. Archived from the original on 21 February 2016. Retrieved 3 February 2016.

2. Kent ME, Romanelli F. Reexamining syphilis: an update on epidemiology, clinical manifestations, and management. Annals of Pharmacotherapy. February 2008; 42 (2):226-36. doi:10.1345/aph.1K086.

3. B'chir Hanzaoui S, Znagui Z, Farah, Bouslama K, Ben Dridi M. A case of papillitis revealing primary syphilis [in French]. Med Mal Infect. 2007; 37:67-68. doi: 10.1016/j.medmal.2006.08.006.

4. Shalaby IA, Dunn JP, Semba RD, Jabs DA. Syphilitic uveitis in human immunodeficiency virus-infected patients. Arch Ophthalmol. 1997; 115:469-473. doi: 10.1001/archopht.1997.01100150471003.

5. Tamesis RR, Foster CS. Ocular syphilis. Ophthalmology. 1990; 97:1281-1287.

6. Kanski JJ. Clinical Ophthalmology. 4th ed., 1999, Oxford, UK, Butterworth-Heinemann, 590-93.

7. Yanoff M, Duker JS, Ophtalmology. $4^{\text {th }}$ ed., 2014.

8. Psomas KC, Brun M, Causse A, Atoui N, Reynes J, Le Moing V. 2012. Efficacy of ceftriaxone and doxycycline in the treatment of early syphilis. Med Mal Infect. 42:1519. doi:10.1016/j.medmal.2011.10.003.

9. Workowski KA, Bolan GA. Centers for Disease Control and Prevention. Sexually transmitted diseases treatment guidelines. MMWR Recommend Rep. 2015; 64(RR-03):1-137. 